\begin{tabular}{|c|c|c|c|c|c|c|}
\hline \multirow{4}{*}{ Impact Factor: } & ISRA (India) & $=3.117$ & SIS (USA) & $=0.912$ & ICV (Poland) & $=6.630$ \\
\hline & ISI (Dubai, UAE & $=0.829$ & РИНЦ (Russia & $=0.156$ & PIF (India) & $=1.940$ \\
\hline & GIF (Australia) & $=0.564$ & ESJI (KZ) & $=8.716$ & IBI (India) & $=4.260$ \\
\hline & JIF & $=1.500$ & SJIF (Morocco & $=5.667$ & OAJI (USA) & $=0.350$ \\
\hline
\end{tabular}

SOI: $\underline{1.1 / \mathrm{TAS}} \quad$ DOI: $10.15863 / \mathrm{TAS}$
International Scientific Journal
Theoretical \& Applied Science
p-ISSN: $2308-4944$ (print) $\quad$ e-ISSN: 2409-0085 (online)
Year: $2019 \quad$ Issue: $06 \quad$ Volume: 74
Published: $25.06 .2019 \quad \underline{\text { http://T-Science.org }}$

SECTION 25. Technologies of materials for the light and textile industry
QR - Issue
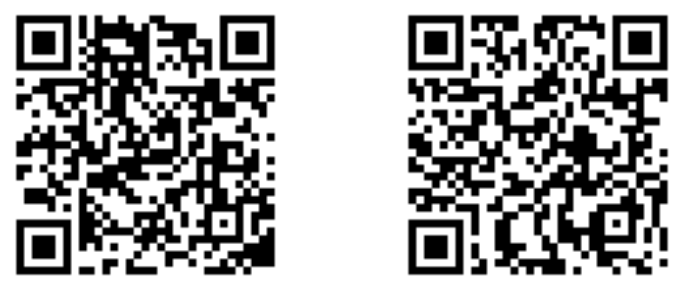

Merab Shalamberidze

Akaki Tsereteli State University

Doctor of Technical Sciences, Professor, Faculty of Technological Engineering, Department of Design and Technology, Kutaisi, Georgia merab.sh@hotmail.com

Zaza Sokhadze

Akaki Tsereteli State University Doctor of Mathematic, Professor, Faculty of Natural Sciences, Department of Mathematics, Kutaisi, Georgia, z.soxadze@gmail.com

Natalia Lomtadze Akaki Tsereteli State University Doctor of Engineering, Ass. Professor, Faculty of Technological Engineering, Department of Design and Technology, Kutaisi, Georgia, lomtadzenato@mail.ru

\title{
CONSTRUCTING THE TRANSVERSE-VERTICAL GEOMETRICAL SHAPE OF THE ORTHOPEDIC SHOE-TREE IN SECTION 0,5D USING THE SOLUTIONS OF SINGULAR DIRICHLET BOUNDARY PROBLEMS
}

\begin{abstract}
The paper presents the results of designing the transverse-vertical section shape of the orthopedic shoe-tree in 0,5D. It is known that from a geometric standpoint, the shoe-tree has a very complicated shape, and its description using mathematical methods of research is a complex and time-consuming process. To solve this problem, the authors of the article used the integral curves of the solutions of singular Dirichlet boundary problems, in particular, for constructing a transverse-vertical shape of the orthopedic shoe-tree in 0,5D. Using the combinations and connections of the segments of integral curves, a transverse-vertical shape was constructed of the orthopedic shoe-tree in 0,5D. This method also allows for changing the shapes on a large scale and with great accuracy to change the shape of the transverse-vertical sections of the orthopedic shoe-tree when shifting from one size to another size. The research results are particularly relevant in the design and production of orthopedic shoe-trees.

Key words: Orthopedic shoe-tree, integral curves, differential equations.

Language: Russian

Citation: Shalamberidze, M. M., Sokhadze, Z. P., \& Lomtadze, N. Z. (2019). Constructing the transversevertical geometrical shape of the orthopedic shoe-tree in section $0,5 \mathrm{~d}$ using the solutions of singular Dirichlet boundary problems. ISJ Theoretical \& Applied Science, 06 (74), 501-504.

Soi: http://s-o-i.org/1.1/TAS-06-74-56 Doi: crostef https://dx.doi.org/10.15863/TAS.2019.06.74.56

\section{ПОСТРОЕНИЕ ПОПЕРЕЧНО-ВЕРТИКАЛЬНОЙ ГЕОМЕТРИЧЕСКОЙ ФОРМЫ ОРТОПЕДИЧЕСКОЙ ОБУВНОЙ КОЛОДКИ В СЕЧЕНИЙ 0,5D С ПОМОЩЬЮ ИНТЕГРАЛЬНЫХ КРИВЫХ РЕШЕНИЙ СИНГУЛЯРНЫХ КРАЕВЫХ ЗАДАЧ ДИРИХЛЕ}

Аннотация: В статье представлень результаты проектирования формы поперечно-вертикального сечения ортопедической обувной колодки на 0,5D. Известно, что обувная колодка с геометрической точки зрения представляет собой очень сложную форму и ее описание с применением математическими
\end{abstract}




\begin{tabular}{|c|c|c|c|c|c|c|}
\hline \multirow{4}{*}{ Impact Factor: } & ISRA (India) & $=3.117$ & SIS (USA) & $=0.912$ & ICV (Poland) & $=6.630$ \\
\hline & ISI (Dubai, UAI & $=0.829$ & РИНЦ (Russia & $=0.156$ & PIF (India) & $=1.940$ \\
\hline & GIF (Australia) & $=0.564$ & ESJI (KZ) & $=8.716$ & IBI (India) & $=4.260$ \\
\hline & JIF & $=1.500$ & SJIF (Morocce & $=5.667$ & OAJI (USA) & $=0.350$ \\
\hline
\end{tabular}

методами исследования является сложным и трудоемким прочессом. Для решения данной проблемы авторы статьи использовали интегральные кривые решений сингулярных краевых задач Дирихле, в частности для построения поперечно-вертикальной формы ортопедической обувной колодки на 0,5D. C помощью комбинаций и соединений отрезков интегральных кривых была построена поперечно-вертикальная форма на 0,5 D ортопедической обувной колодки. Данный метод дает также возможность в широком масштабе и с большой точностью менять формы поперечно-вертикальных сечений ортопедической обувной колодки при переходе от одного размера на другой размер. Результатьл исследований особенно актуальны при проектировании и в производстве ортопедчческой обувной колодки.

Ключевые слова: Ортопедическая обувная колодка, интегральные кривые, дифференциильные уравнения.

The work was executed with the financial support of Shota Rustaveli National Science Foundation, grant FR № 217386.

Работа выполнена при финансовой поддержке национального научного фонда Грузии имени Шота Руставели, грант FR № 217386.

\section{Введение.}

В обувной промышленности большое внимание уделяется вопросу проектирования ортопедической обувной колодки. Известно, что форма обувной колодки с геометрической точки зрения является сложным и ее описание математическими методами исследования является довольно долгим и трудоемким процессом. В процессе проектирования обувной колодки в обязательном порядке учитываются антропометрические данные стопы, такие, как размеры и формы. Авторами статьи были исследованы педографические, антропометрические и тензометрические данные стоп пациентов. Создана база данных, в которой индивидуально зафиксированы патологические отклонения стоп пациентов.

Для производства сложной ортопедической обуви проектируют такие специальные колодки, которые учитывают все патологические отклонения деформированной стопы. Только в этом случае образуется комфортное внутри обувное пространство для нормального функционирования стоп с патологическими отклонениями.

Алгоритмы, описывающие геометрическую форму обувной колодки, рассмотрены в работах широкого круга ученных [1-5]. Для описания геометрической формы поперечно-вертикальных сечений обувной колодки они использовали следующие методы исследования: радиус графический, биквадратный сплайн, бикубический интерполярный сплайн и др. Вышеуказанные методы исследования требуют большого времени в процессе проектирования, а также характеризуются некоторыми отклонениями в достоверности.

\section{Методы исследования.}

Для системного описания поперечновертикальных сечений ортопедической обувной колодки авторы статьи применили интегральные кривые решений сингулярных краевых задач Дирихле [6, 95-127]. С помощью выше указанного математического метода исследования нами были построены формы поперечно-вертикальных сечений ортопедической обувной колодки на $0,18 \mathrm{D}, 0,62 \mathrm{D}$ и $0,78 \mathrm{D}[7,27-32 ; 8,23-31,9,122-126$, $10,17-21]$. Аналогичным методом была построена геометрическая форма поперечно-вертикального сечения ортопедической обувной колодки на $0,5 \mathrm{D}$.

\section{Результаты и их обсуждения.}

В процессе исследования преобразовали биомеханические характеристики деформированных стоп и на этой основе определяли кривые поперечно-вертикального сечения колодки на $0,5 \mathrm{D}$.

На рис. 1 представлен поперечновертикальное сечение ортопедической обувной колодки на $0,5 \mathrm{D}$. 


\begin{tabular}{|c|c|c|c|c|c|c|}
\hline \multirow{4}{*}{ Impact Factor: } & ISRA (India) & $=3.117$ & SIS (USA) & $=0.912$ & ICV (Poland) & $=6.630$ \\
\hline & ISI (Dubai, UAI & $=0.829$ & РИНЦ (Russia & $=0.156$ & PIF (India) & $=1.940$ \\
\hline & GIF (Australia) & $=0.564$ & ESJI (KZ) & $=8.716$ & IBI (India) & $=4.260$ \\
\hline & JIF & $=1.500$ & SJIF (Morocce & $=5.667$ & OAJI (USA) & $=0.350$ \\
\hline
\end{tabular}

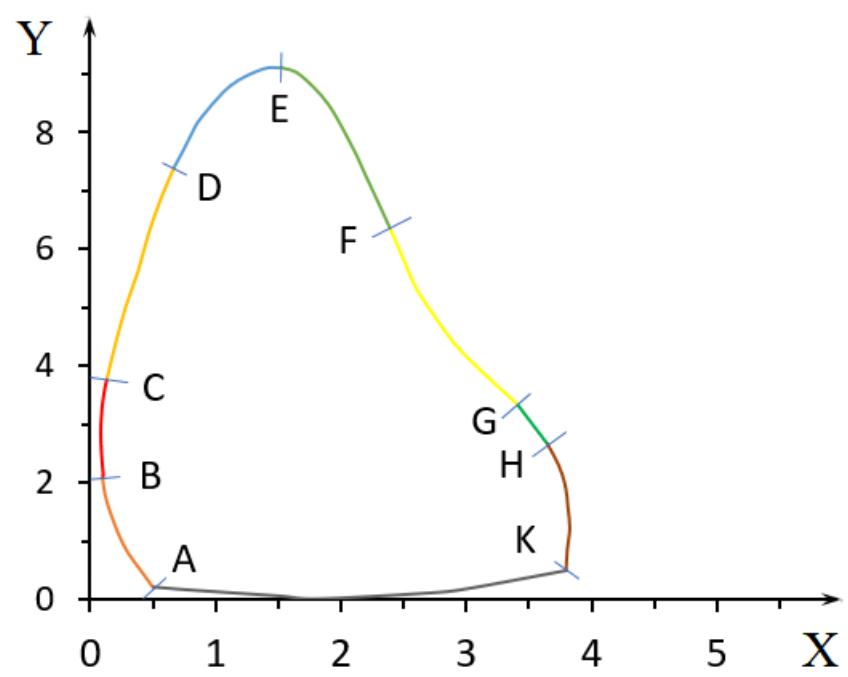

Рис. 1. Поперечно-вертикальное сечение ортопедической обувной колодки на 0,5D.

Для построения поперечено-вертикального сечения ортопедической обувной колодки на $0,5 \mathrm{D}$ ее предварительно разделили на девять частей. Кривых пронумерованных девяти отрезков описывали с помощью дифференциальных уравнений. С помощью интегральных кривых решений сингулярных краевых задач Дирихле выбирали те девять отрезков, которые идентично соответствуют геометрической форме поперечновертикального сечения на $0,5 \mathrm{D}$ ортопедической обувной колодки, в частности:

I - кривая $\mathrm{AB}$ соответствует той части решения уравнения $u(t)=\left(-\frac{1}{3}-\frac{1}{3} c\right) t+\frac{1}{3} c \frac{1}{t^{2}}+$ $\frac{2}{3} t+\frac{t^{2}}{2}+\frac{t^{4}}{6}$, для которой $\mathrm{c}=0$, с множеством $[-2,4 ; 1.8] \times[1.8 ;-0.5]$;

II - кривая $\mathrm{AB}$ соответствует той части решения уравнения $u(t)=\left(-\frac{1}{3}-\frac{1}{3} c\right) t+\frac{1}{3} c \frac{1}{t^{2}}+$ $\frac{2}{3} t+\frac{t^{2}}{2}+\frac{t^{4}}{6}, \quad$ для $\quad$ которой $\quad \mathrm{c}=0, \quad \mathrm{c}$ множеством[1.7: 2.3] × [1.8; 2.9];

III - кривая CD соответствует той части решения уравнения $u(t)=\left(-\frac{1}{3}-\frac{1}{3} c\right) t+\frac{1}{3} c \frac{1}{t^{2}}+$ $\frac{2}{3} t+\frac{t^{2}}{2}+\frac{t^{4}}{6}$, для которой $\mathrm{c}=5$, с множеством $[-2.8 ;-2.1] \times[9.1 ; 4.2]$;

IV - кривая DE соответствует той части решения уравнения $u(t)=\frac{t^{3}}{2}+\frac{1}{3} c t^{-2}-(1-$ $\left.\frac{1}{3} c\right) t+\frac{1}{2}$, для которой $\mathrm{c}=3$, с множеством $[-5.1 ;-1.6] \times[-2.1 ;-0.9]$;

$\mathrm{V}$ - кривая EF соответствует той части решения уравнения $u(t)=\frac{t^{3}}{2}+\frac{1}{3} c t^{-2}-(1-$ $\left.\frac{1}{3} c\right) t+\frac{1}{2}$, для которой $\mathrm{c}=5, \quad$ с множеством $[-0,9 ;-0,1] \times[0.1 ;-6.1]$;
VI - кривая FG соответствует той части решения уравнения $u(t)=\frac{t^{3}}{2}+\frac{1}{3} c t^{-2}-(1-$ $\left.\frac{1}{3} c\right) t+\frac{1}{2}$, для которой $\mathrm{c}=0, \mathrm{c}$ множеством $[-2.7 ;-1.6] \times[-7 ; 0.1]$;

VII - кривая GH соответствует той части решения уравнения $u(t)=\left(-\frac{1}{3}-\frac{1}{3} c\right) t+\frac{1}{3} c \frac{1}{t^{2}}+$ $\frac{2}{3} t+\frac{t^{2}}{2}+\frac{t^{4}}{6}$, для которой $\mathrm{c}=2$, с множеством $[-1.9 ;-1.8] \times[2.1 ; 3.2]$;

VIII - кривая HK соответствует той части решения уравнения $u(t)=\left(-\frac{1}{3}-\frac{1}{3} c\right) t+\frac{1}{3} c \frac{1}{t^{2}}+$ $\frac{2}{3} t+\frac{t^{2}}{2}+\frac{t^{4}}{6}$, для которой $\mathrm{c}=1, \mathrm{c}$ множеством $[-1 ;-0.4] \times[0.1 ; 2.7]$;

IX - кривая KA соответствует той части решения уравнения $u(t)=\frac{t^{3}}{2}+\frac{1}{3} c t^{-2}-(1-$ $\left.\frac{1}{3} c\right) t+\frac{1}{2}$, для которой $\mathrm{c}=2, \quad$ с множеством $[1.2 ; 2.8] \times[0.8 ; 14,8]$.

\section{Выводы.}

Таким образом, на основе базы данных патологических отклонении стоп пациентов и с помощью интегральных кривых решений сингулярных краевых задач Дирихле, построено поперечно-вертикальное сечение на 0,5D ортопедической обувной колодки.

С использованием вышеуказанного метода исследования можно построить любые формы поперечно-вертикальных сечений ортопедической обувной колодки. Также, данный метод дает возможность менять формы поперечно-вертикальных сечений при переходе от одного размера колодки на смежный размер колодки. Использование данного математического метода исследования в процессе проектирования ортопедической колодки 


\begin{tabular}{|c|c|c|c|c|c|c|}
\hline \multirow{4}{*}{ Impact Factor: } & ISRA (India) & $=3.117$ & SIS (USA) & $=0.912$ & ICV (Poland) & $=6.630$ \\
\hline & ISI (Dubai, UAE & $=0.829$ & РИНЦ (Russia) & $=0.156$ & PIF (India) & $=1.940$ \\
\hline & GIF (Australia) & $=0.564$ & ESJI (KZ) & $=8.716$ & IBI (India) & $=4.260$ \\
\hline & JIF & $=1.500$ & SJIF (Morocco) & $=5.667$ & OAJI (USA) & $=0.350$ \\
\hline
\end{tabular}

является актуальным, особенно для пациентов с деформированными и патологическими стопами.

\section{References:}

1. Fukin, V. A. (1980). Radiusograficheskiy metod konstruirovaniya vnutrenney formy obuvi. (p.381). Moscow: Legkaya industriya.

2. Fukin, V. A., Kostyleva, V. V., Lyba, V. P. (1987). Proektirovanie obuvnykh kolodok. (p.268). Moscow: Legprombytizdat.

3. Fukin, V. A. (2000). Teoreticheskie osnovy proektirovaniya vnutrenney formy obuvi. 2izdanie per. i dop. (p.356). Moscow: MGUDT.

4. Kiselev, S. Y. (2004). Avtomatizirovannoe proektirovanie i izgotovlenie tekhnologicheskoy osnastki dlya proizvodstva obuvi $i$ protezoortopedicheskikh izdelii. Diss. d-ra tekh. nauk. (p.392). Moscow: MGUDT.

5. Zamarashkin, K. N. (2004). Matematicheskie metody proektirovaniya obuvi $i$ konstruirovaniya tekhnologicheskoy osnastki. (p.312). SPB.: SPGUTD.

6. Rachunkova, I., Spielaurer, A., Stanek, S., \& Weinmuler, E. B. (2013). The sttucture of a set of positive solutions to Dirichlet BVPs with time and space singularities. Georgian Mathematical Journal, №1, pp. 95-127.

7. Shalamberidze, M., Sokhadze, Z., \& Tatvidze, M. (2018). Construction of the Transverse-
Vertical Shapes of the Orthopedic Boot-Tree by Means of the Solution to Singular Dirichlet Boundary Value Problem. Bulleten of the Georgian National Academy of Sciences, Vol. 12, №1, pp. 27-32.

8. Shalamberidze, M., Sokhadze, Z., Tatvidze, M. (2018). Constructing the main transversevertical cross-sections of the orthopedic shoe boot tree by means of the integral curves. Bulleten of the Georgian National Academy of Sciences, Vol. 12, №3, pp. 23-31.

9. Shalamberidze, M., Sokhadze, Z. (2018). Constructing a shape of orthopedic boot-tree print by means of the solution to differential equation with deviating argument. Philadelphia USA. International Scientific Journal. Theoretical \& Applied Science, vol. 61, №5, pp. 122-126.

10. Shalamberidze, M., Sokhadze, Z., Tatvidze, M. (2019). Construction of the Orthopedic BootTree Print and Main Longitudinal-Vertical Section by Means of the Solution of Differential Equations. Bulleten of the Georgian National Academy of Sciences, Vol. 13, №2, pp. 17-21. 